\title{
ARTIFICIAL INTELLIGENCE BASED CONTROL APPROACH FOR MEMBRANE BIOREACTOR IN SEWAGE WATER TREATMENT
}

\author{
D.Deena $^{1}$, J.Sureshkumar ${ }^{2}$ \\ ${ }^{1}$ Assistant Professor, Department of Electrical and Electronics Engineering, United Institute of technology Coimbatore \\ ${ }^{2}$ Assistant Professor, Department of Civil Engineering, Sree Sakthi Engineering College, Karamadai, Coimbatore
}

\begin{abstract}
Membrane bioreactors (MBR) for wastewater treatment, which have increasingly been employed for the last 15 years and which play an important role in wastewater treatment. It is the continuation of the fundamental contribution of sewage water treatment, which is extended and complemented in various aspects. In this project an advanced control approach is made to bring desired purification for sewage water. The major challenge in controlling MBR is the large amount of uncertainty present in the process models, in the unknown inflow conditions, and in the limited measurement information. Hence, controller development is driven by the necessity to structure uncertainty, to attenuate its harmful effects, and to exploit it wherever possible. Such a control method is model based predictive controller. The frame work for the model-based control of membrane bioreactors (MBR) is developed, which aims at the economical optimization of MBR operation
\end{abstract}

Keywords: MBR, NMPC, Optimization. $* * *$

\section{INTRODUCTION}

Water is a global resource indispensable for life on earth. Its responsible and sustainable use and reuse is a major challenge of the 21st century. The increasing world population and industrialization lead to a rising demand for potable and process water, and in many areas existing supplies are diminishing at critical rates. Untreated wastewater threatens intact biological systems by introducing large amounts of nutrients, toxic or endocrines species, heavy metals and other harmful components. For these reasons efficient water treatment and reuse have become decisive social and economical issues.

In many countries legal limits on the effluent concentrations of selected components are tightened, e. g. by the European Water Framework Directive issued in 2000. Strict effluent constraints however together with increasing wastewater loads demand efficient treatment processes. At the same time the increasing privatization of wastewater treatment facilities requires a stronger focus on their economic performance. This context provides the motivation for the control approach presented here. MBR combine classical biological wastewater treatment with subsequent membrane filtration. The membrane unit separates the biomass of the biological treatment from the water. MBR offer high effluent quality, reliable biomass separation and small space requirements.

These properties make them an appealing alternative especially when effluent constraints are tight, when space is limited and when existing plants need to be upgraded.
In general, however MBR operating costs are higher than those of conventional wastewater treatment plants (WWTP), which employ sedimentation basins for the biomass separation a large potential to increase the economic feasibility of MBR lies in the improvement of their operational policy. Until today only simple control strategies have been employed Advanced control approaches frequently used in the chemical process industry have not been applied to MBR due to the large uncertainty in the biological and the filtration processes, in the inflow prediction, and in the limited measurement information.

While this is not different from the obstacles in regular WWTP operation, the increased complexity of MBR requires efficient online control to exploit their full potential. Hence, this project focuses on the process control of MBR. It aims at bringing advanced approaches from many research areas as e.g. control and optimization together to provide a capable, flexible and generic control architecture which takes the characteristics and peculiarities of MBR and MBR operation into account. Due to the process complexity model-based control approaches are proposed. Time and unit scale separation are performed to obtain sub problems of lower complexity for different disturbance dynamics and for both the biology and the membrane system. For each of them suitable models, problem formulations, and efficient solution algorithms need to be formulated. The coordination between the sub problems on different time scales and between the units must be considered. 


\section{PROCESS DESCRIPTION OF MEMBRANE BIOREACTOR}

Wastewater treatment in day today life is a pressing need in view of decreasing surface water resources and falling groundwater levels. Increasing water consumption mandates the intensified reuse of water and has leaded to increasingly strict legislative limits on effluent concentrations. In consequence, wastewater treatment has become a large industry with high annual product volume and financial investments (Gray, 2004).

Economical pressure calls for efficient process solutions. Membrane bioreactors (MBR) are one promising technology in this context (Wintgens et al., 2005). MBR combine two technologies to efficiently and reliably purify wastewater up to high quality standards: traditional biological treatment to remove carbon, nitrate, phosphorus compounds and subsequent membrane filtration to separate the biomass and other particulate matter from the purified water.

Although each MBR has its unique properties, there are main design features which are common to most plants. Wastewater enters the plant and is temporarily stored in a storm water tank to level out peaks in the inflow rate and concentrations.

Mechanical units such as sieves and sand filters remove coarse particulate matter and fat. The subsequent biology is typically divided into two zones, the denitrification and nitrification which can each comprise one or several basins.

Here the biological reactions take place, turning the contaminants into biomass, carbon dioxide and gaseous nitrogen. The nitrification basin is aerated to provide dissolved oxygen. Sludge can be withdrawn from the bottom of the basin. A recirculation stream transports sludge from the nitrification back to the denitrification. Finally, a membrane unit separates the inert as well as the biologically active particulate matter from the product water. The product water is withdrawn, while the particulate matter is retained in the system.

In the configuration shown here the membrane unit requires an additional air stream, whose function is discussed later. In the following sections, the main process are units storm water tank, mechanical cleaning, biology, and membrane system are discussed.

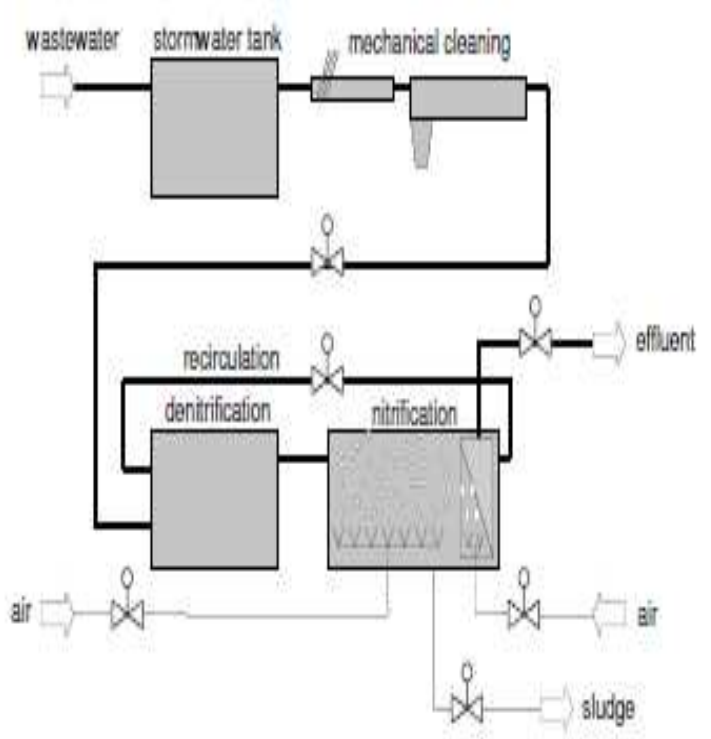

Fig 1 Process Diagram for MBR

Finally, the MBR process as a whole is considered, highlighting its characteristics in comparison to conventional wastewater treatment plants (WWTP) and the couplings between the biological and the membrane system, the development of advanced model-based control approaches for MBR is motivated.

\section{MODEL-BASED CONTROL OF MBR}

Efficient control of membrane bioreactors (MBR) is a challenging task. The uncertainty in the process kinetics and mechanisms, the stiff process dynamics, the lack of meaningful and reliable online measurements, the highly dynamic and uncertain inflow and the hardly understood relationships between the biology and the filtration system pose significant difficulties.

The objective of advanced control approaches for MBR is to realize a reliable and cost efficient purification of wastewater up to specified standards in spite of these challenges.

State-of-the-art of MBR control is based on the experience in controlling conventional biological treatment plants. The MBR biology is controlled in the same fashion as regular biology, though partly at different set points, e. g. at higher biomass concentrations. Typical control schemes are PID type control of the dissolved oxygen or ammonia concentrations.

The membrane system is operated according to the set points suggested by the manufacturer or by experienced operators. Fixed set points for the controlled variables are common, with some variation to meet the required net flux[6]. 
The main challenge is to deal on the one hand with the high uncertainty and stiff dynamics of the two complex systems and on the other hand with their interaction. Given this situation together with the high number of controlled and manipulated variables, constraints and objectives a model-based approach to the problem seems inevitable.

Model-based control approaches offer the advantage that they can accommodate process and input constraints, Can incorporate economic objectives in addition to set point control, Can deal with high process complexity, and Consider past, present, and predicted future process behaviour.

The process dynamics are very stiff and recalling the cyclic behaviour of the filtration system there are considerable structural differences between the operation of the biology and of the membrane system.

It is therefore expected to be highly impractical or even impossible to describe the entire system with one model so an alternative approach is to divide the MBR control problem into several sub problems, which can be approached with tailored methods[6]. The key idea of the latter is to separate the disturbance and process dynamics according to their relevant time scales and to assign them to different interrelated operational layers.

\section{SCHEMATIC CONTROLLER FLOW DESCRIPTION FOR THE PROCESS PLANT}

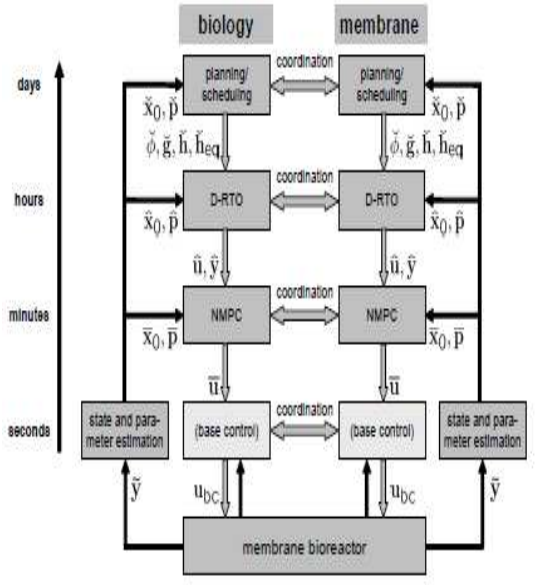

Fig. 2 Schematic Controller Flow Diagram of Process Plant

On the left and right of Fig.2 the control system for the biology and the control system related to the membrane unit are depicted respectively. Various coordinators are suggested between the two systems. The vertical axis represents the time scales on which the control layers operate, starting with the slowest dynamics from above.
The operational layers depicted in Fig.2 are planning/scheduling, dynamic real-time optimization (D-RTO), non-linear model predictive control (NMPC), base control, and state and parameter estimation.

The base control and the estimation layers are connected to the controlled unit, which can be the real plant or a plant simulation layer [2]. On the planning/scheduling layer fundamental planning decisions about the operation of the plant are made (Shah, 1998). This layer determines the objectives ǒ as well as the path inequality constraints $\breve{g}$ and equality (heq) and inequality $(\hat{\mathrm{h}})$ endpoint constraints of process operation.

The objectives may e. g. include economical and ecological objectives, and the constraints may refer to effluent concentration limits or tank holdups. Based on the information from the planning/scheduling layer, the optimal trajectories of the inputs $\breve{u}$ and outputs are computed on the D-RTO layer by solving a non-linear, constrained, dynamic optimization problem.

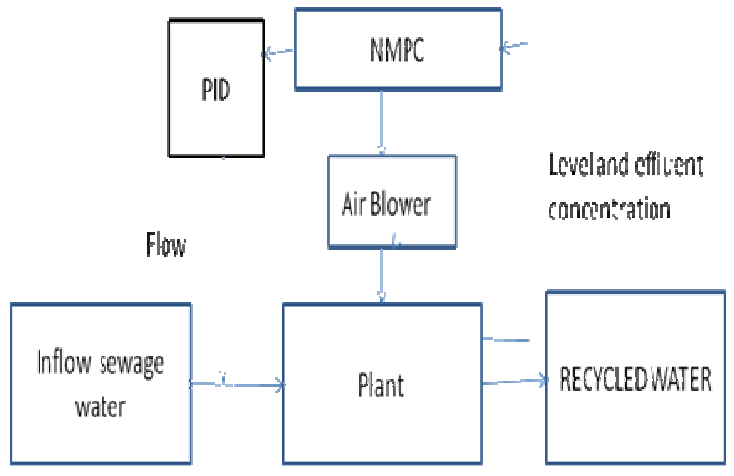

Fig.3 Proposed method block involving NMPC system

Accordingly, complex models with good predictive capabilities have to be employed. If the inputs ŭ were implemented as such, plant model mismatch and disturbances would inevitably lead to divergence of the optimal ( $\hat{y})$ and the real (y) output trajectories. Typically the computation of the D-RTO problem is too demanding to be performed on the time scale of these disturbances. Hence the NMPC layer is required for adequately fast responses.

In NMPC, an optimization problem is solved again, but now the objective is not an economical one anymore, but the minimization of the difference between the optimal and the real input and output trajectories. The model employed on the NMPC layer may be of less complexity, as the prediction horizon is smaller and the model only needs to be accurate in the subspace around the real and optimal trajectories.

The NMPC layer provides corrected inputs $u$. If the process deviates too far from the optimal solution, the NMPC layer will 
not be able to realize the desired behaviour anymore, therefore periodic or intelligently triggered updates of the D-RTO trajectory are required. However, MBR plants possess unique features, which allow simplifying the concept at some points and requiring extensions at others.

With respect to the biology, the planning/scheduling and the DRTO layers have been merged into a dynamic predictive scheduling layer. On this new layer the scheduling of operational strategies (objectives and constraints) is solved simultaneously with the D-RTO problem.

In this project, a framework for the model-based control of membrane bioreactors (MBR) is developed, which aims at the economical optimization of MBR operation [2]. It is the continuation of the fundamental contribution of Cruse (2006), which is extended and complemented in various aspects.

Hence, controller development is driven by the necessity to structure uncertainty, to attenuate its harmful effects, and to exploit it wherever possible. Planning is understood as strategic decision making for the operation of one or several plants e. $g$. based on long-term predictions of market prizes or customer demand.

Scheduling on the other hand is concerned with the fulfilment of fairly specific production requirements on a shorter time horizon (Shah, 1998). In both approaches, discrete decisions are made concerning e.g. investments, the employment of certain units or pipelines or the sequencing of production campaigns.

In D-RTO, optimal set points or set point trajectories for the manipulated and the controlled variables of the associated process are computed. Obviously planning, scheduling, and set point optimization are interrelated, while the degree of interdependency is specific to each considered process. Uncertainty is a major challenge to all scheduling approaches. It can be shown that nominally optimal solutions yield suboptimal or even infeasible solutions when realized under uncertainty. Uncertainty is mostly related to the model structure, the model parameters, and disturbances.

\section{SIMULATION RESULTS AND DISCUSSION}

The Extended Kalman Filter is employed to provide initial state estimates at each LTV-MPC optimization. It is assumed that in each basin the concentrations of oxygen, ammonia, nitrate, alkalinity, solids, chemical oxygen demand (COD), filtered COD, and biological oxygen demand (BOD) are measurable and that the measurements of the inflow rate and inflow concentrations are perfect. Gaseous nitrogen is neither measurable nor observable, but since it does not influence any reaction its estimate is not relevant for the process prediction [1]. A measurement noise of $2 \%$ is introduced, and no process noise is assumed.
No constraints on the outputs, inputs, or states are formulated. It is constant at $520 \mathrm{~m} 3 / \mathrm{d}$. In the same diagram the input trajectory as computed by the NMPC (LTV-MPC) layer for the undisturbed process is depicted. It stays close to the reference trajectory. The deviations are caused by the variations in the state estimates [1].

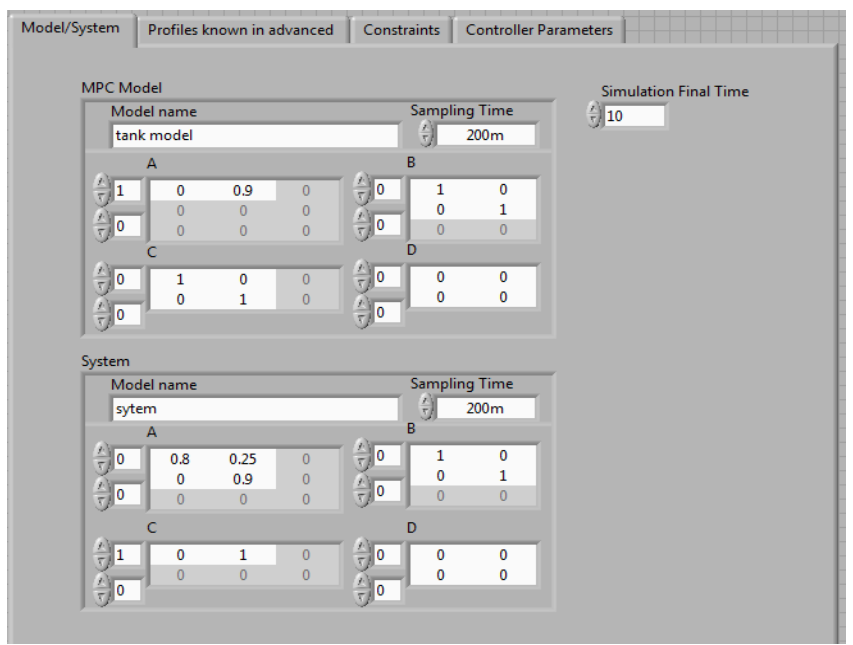

Fig. 4 simulation result of modelling controller using labview.

A measurement noise of $2 \%$ is introduced, and no process noise is assumed. No constraints on the outputs, inputs, or states are formulated. The three weighting matrices $\mathrm{Q}, \mathrm{R}$, and $\mathrm{S}$ was formulated. The weighting matrix $\mathrm{R}$, which penalizes input deviations, is set to zero. The matrices $\mathrm{Q}$ and $\mathrm{S}$ are set to the identity matrix. The inputs $u$ are scaled to values around $1[1]$. They comprise the permeate flow rate $\mathrm{ZP}$, the aeration kla, the recycle flow rate ZND, and the sludge withdrawal ZS, The reference trajectory of the permeate flow rate $\mathrm{ZP}$ is shown in figure. It is constant at $520 \mathrm{~m} 3 / \mathrm{d}$. In the same diagram the input trajectory as computed by the NMPC (LTV-MPC) layer for the undisturbed process is depicted

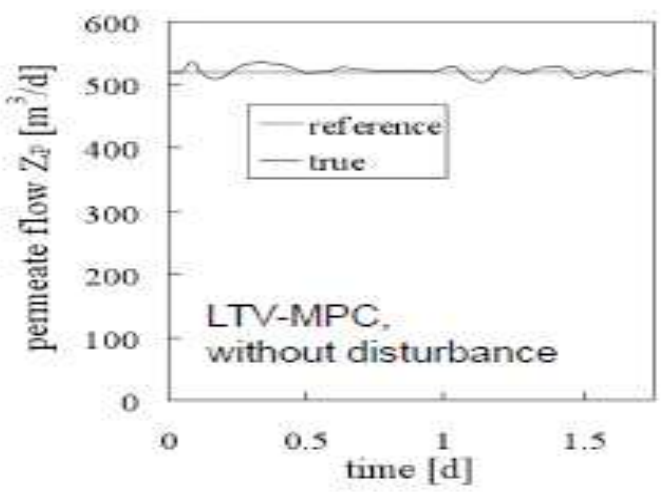

Fig.5 a Reference profiles of the DPS layer and LTV-MPC profiles for the permeate flow $\mathrm{ZP}$ for the undisturbed process. 


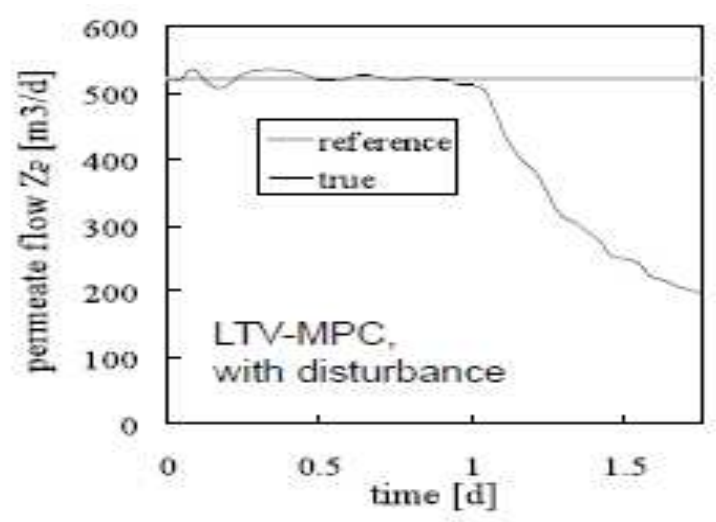

Fig.5 b Reference profiles of the DPS layer and LTV-MPC profiles for the permeate flow $\mathrm{ZP}$ for the disturbed process.

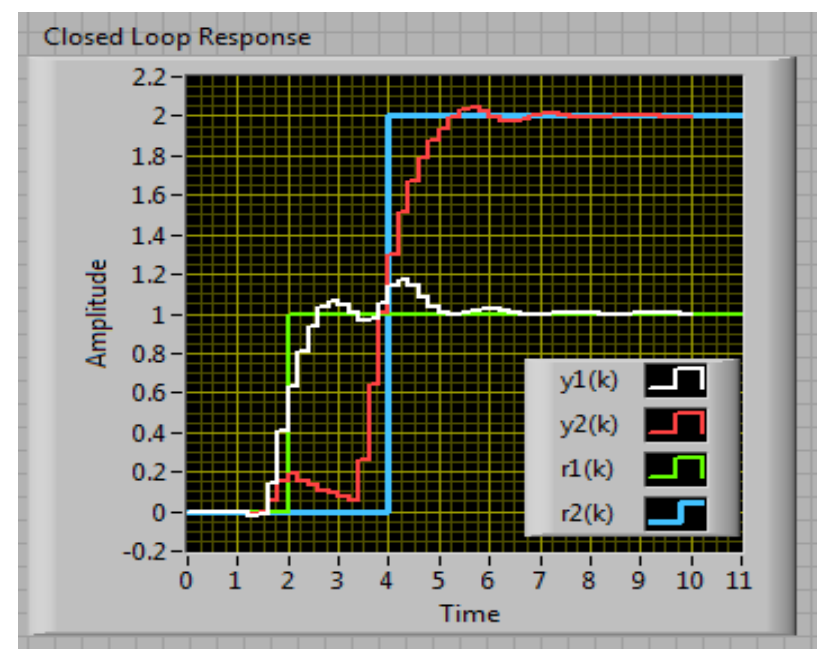

Fig. 6 Aeration and flow control output using labview

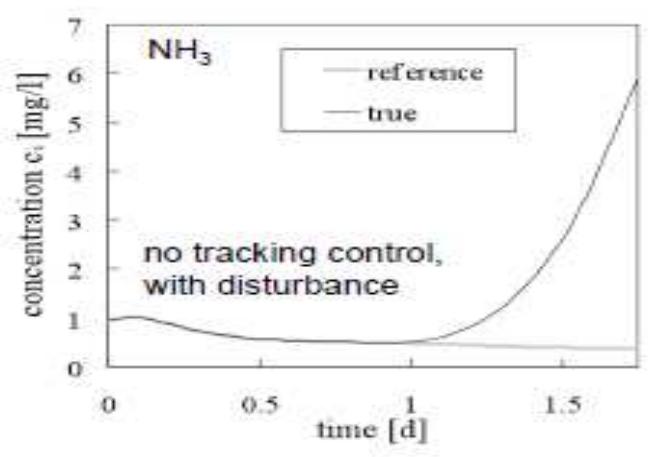

Fig. 7 Reference profiles of the DPS layer and obtained profiles for the effluent concentrations of ammonia $\mathrm{cN}, \mathrm{SNH}$ (top) for the process with no tracking control
The LTV-MPC trajectory of the permeate flow rate is shown on the right of Fig3b. During the first day the concentration profiles and the permeate flow rate match the reference profiles closely due to the absence of disturbances. When the disturbance appears after approximately 1 day, the permeate flow is gradually decreased. This increases the hydraulic residence time of the system and enables the biology to treat the increased amount of ammonia and nitrate.

The other input variables are adapted as well (not shown). As a result, the profiles of the ammonia and nitrate concentrations are forced back to their reference profiles. The ammonia concentration profile exhibits a noticeable deviation from the reference profile when the disturbance occurs, but the deviation is small compared to the offset observed in the uncontrolled case (Fig.4).

The tracking of the reference trajectories is nearly perfect when perfect knowledge of the states is assumed (not shown). The offset observed is hence rather related to the challenging state estimation task than the LTV-MPC controller

\section{CONCLUSIONS}

This Non-Linear Model based Predictive Controller methodology to MBR in sewage water treatment brings an efficient controlling strategy to the entire plant by automatic rescheduling of their set points for unknown inflow conditions together by optimizing set point values.

This set point value should match the bioreactor operational strategy that may provide a capable and flexible operation. This controller operation is expected to get more efficient filtration of sewage water which can be reused.

\section{REFERENCES}

[1] An integrated process of jet loop bioreactor and ultrafiltration membrane used for wastewater treatment in Shanghai, China

[2] Naundordf E A, Subramanian D, Pabiger Nl. Biological treatment of wastewater in the compact reactor. Chem Eng Process, 1995, 19: 229-234.

[3] Xiufen Li, Fangshu Gao, Zhaozhe Hua, et.a!. Treatment of synthetic wastewater by a novel MBR with granular sludge developed for controlling membrane fouling. Separation and Purification Technology, 2005 (46): 19-25

[4] Xiang Zheng, Junxin Liu. Dyeing and printing wastewater treatment using a membrane bioreactor with a gravity drain. Desalination, 2006(190): 277-286

[5] Guglielmi, G.: 2002, Membrane bioreactors for municipal wastewater treatment, $\mathrm{PhD}$ thesis, Universit`a di Trento, Italy. 
[6] Gujer, W., Henze, M., Mino, T. and van Loosdrecht, M.: 1999, Activated sludge model no.3, Wat. Sci. Tech. 39(1), 183-193.

[7] Hamaker, H. C.: 1937, The London-Van-der-WaalsAttraction between sperical particles.

[8] H. Henze, C.P.L. Jr. Grady, W. Gujer, G.V.R. Marais, 1987, T. Matsuo, A general model for single-sludge Wastewater Treatmant systems, 1987, Wat. Res., 21(5), 505-515.

[9] I. Takaks, G.G. Patry, D. Nolasco, 1991, A dynamic model of the clarification thickening process, Wat. Res., 25(10), 1263-1271.

[10] M.V. Cristea, S.P. Agachi, V. Marinoiu, 2003, Simulation and Model Predictive Control of a UOP Fluid Catalytic Cracking Unit, Chem. Eng. and Proc., 42, 67-91.

[11] M. Yong, P. Yongzhen, U. Jeppsson, 2006, Dynamic evaluation of integrated control strategies for enhanced nitrogen removal in activated sludge process, Contrl. Eng. Prac., 14, 1269-1278.

[12] Nikolic V, Fuzzy and neuro-fuzzy systems in problems of process control and modeling: possibilities and some aspects of application, Journal of Process Technology, Vol. XXV, No. 3, Belgrade, pp. 164-167, 1999.

[13] Eva L. Decker und Ralf Reski : Current achievements in the production

[14] of complex biopharmaceuticals with moss bioreactor. Bioprocess and Biosystems Engineering, (2008) 31, 39

[15] Jong-Sang Park, Kyung-Min Yeon, Chung-Hak Lee. Hydrodynamics and microbial physiology affecting performance of a new MBR, membrane-coupled highperformance compact reactor [J]. Desalination.(2005).

[16] Kyung-Min Yeon, Jong-Sang Park, Chung-Hak Lee. Membrane coupled high-performance compact reactor: A new MBR system for advanced waste water treatment $[\mathrm{J}]$. Water Research (2005).

[17] Lopez A, Lazaro N, Marques AM. The interphase technique: a simple method of cell immobilization in gel-beads. J Microbiol Methods,(1997),

[18] Naundordf E A, Subramanian D, PabigerNl. Biological treatment of wastewater in the compact reactor.ChemEng Process, (1995), 19: 229-234.

[19] Peinado PA, Moreno JJ, Villaba JM, Gonzalez-Reyes JA, Ortega JM, Mauricio JC. A new immobilization method and their applications. Enzyme

[20] Microb Tech, (2006), 40:79-84.Wildhagen H, Schneid F, Geiben Al. Pilot test of biological treatment of brewery effluent using HCR system at Gilde Brewery in Hanover[J] Chemeng process.(1992). 